$$
\text { استخدام وسائل التواصل الاجتماعي للحفاظ على التراث الثقافي المغربي }
$$

\title{
Using social media to preserve the Moroccan cultural heritage
}

رحاب عصام حسنى محمد ، باحثة دكتوراه ، كلية الدراسات الافريقية العليا ، جامعة القاهرة ، مصر ، samaelrehab@gmail.com

تعد وسائل التواصل الاجتماعي من أهم وسائل الاتصال في عالمنا الحديث ، فأصبح لها تأثير على الحياة الاجتماعية والثقافية والاقتصادية والسياسية ليس علي الفرد فقط وإنما على المجتمع ككل ، وتعد المغرب من الدول الزاخرة بالتراث الثقافي ، لذا كان من المهم الحفاظ على الهوية المغربية دون طمس ثقافتها الأصلية عن طريق تلك الوسائل من أجل ترسيخ الهوية دون التأثر بالثقافات الغربية . لذا يتمحور البحث حول "استخدام وسائل التواصل الاجتماعي للحفاظ على التراث الثقافي المغربي" ، خاصة لكل من موقع الفيسبوك وقنوات اليوتيوب ، فهم من الوسائل الفعالة في الوقت الحالي ، ويسهل استخدهم ويمكن عن طريقهم التوعية والحفاظ بالتراث الثقافي ، وسيتم ذلك عن طريق عرض لتلك الوسائل التي تداول موضوعات ذات صلة بالتراث الثقافي المغربي وأهم المواضيع المتداولة في تلك الوسائل والمتضمنة التراث الثقافي المغربي ، من خلال تحليل مضمون لبعض نماذج وسائل التواصل الاجتماعي للتراث الثقافي المغربي والمتخصصة من المعمار المغربي واللباس التقليدي والمطبخ المغربي وكنلك حكايات شعبية عن التراث المغربي ، ومن تلك الوسائل: صفحة الفيسبوك "المغرب" ، وقنوات اليوتيوب: "الفراشة" ، "عالم الأناقة والجمال المغربي" ، "تكهة مغربية" ، "المطبخ المغببي مع ربيعة" ، "قناة هبة للطبخ المغربي" ، "حجايات شعبية باللسان المغربي الدارج" ، "حكايات شعبية ما احلاها" . الكلمات المفتاحية : وسائل التواصل الاجتماعي - التراث الثقافي 
يلعب الانترنت دور مهم في حياتتا اليومية ، وأصبح لوسائل التواصل الاجتماعي دور رئيسي بها ، فلقد حازت على اهتمام الكثير من المجتمعات فهي عبارة عن مواقع اجتماعية يتواصل ويتفاعل الافراد من خلالها كذلك يتاقلون الخبرات عن طريقها ، ومع التطور التكنولوجي أصبحت تلك الوسائل أسرع في الانتشار وأرخص في التكلفة من وسائل الاعلام التقليدية الأخرى فعن طريق الاجهزة اللوحية التي يحملها غالبية أفراد المجتمع سهلت عليهم مشاهدة ما يهتم به في أي وقت وأي مكان ، ومع انتثار مواقع التواصل الاجتماعي من "تويتر وفيسبوك ويوتيوب وغيرها..." أصبحت تلك المواقع منصات أساسية تعرض كل ما هو مختلف وجديد ، مما أتاحت الفرصة للاطلاع على الثقافات المختلفة للمجتمات من عادات وتقاليد ومورثها الثقافي فبذلك أصبحت وسائل التواصل الاجتماعي ذات أهمية للترويج عن التراث الثقافي للمجتعات ، فهي تعكس ماضي المجتمعات لتستمر في عيش حاضرها وبناءً مستقبلها. وتزخر المغرب بتراث ثقافي غني ومتتوع ، فهي تنحدر من امتزاج عدة روافد منها : الأفريقي ، الأمازيغي ، الصحراوي ، العربي ، والاندلسي .. هذا ما يجعل طابع التتوع يطغي على تراثها الغني جداً . ونظراً لها يضمه المغرب من قيم وعادات وتقاليد متوارثة من الاجيال السابقة والتي تعبر عن هويتهم بما أوجب الحفاظ عليها من الاندثار عن طريق استخدام وسائل التواصل الاجتماعي لتوعية المجتمع المغربي بأهميته وضرورة التمسك بهويته لمواجهة المتغيرات الحديثة والتي جعلت الفرد يتبني ثقافات دخيلة عن ثقافته الاصلية مما قد يؤثر عليه بالابتعاد عنها ونسيان ثقافته المحلية . وسيعتمد هذا البحث علي أداة تحليل المضمون لبعض النماذج المغربية لوسائل التواصل الاجتماعي والتي تتناول التراث الثقافي المغربي من خلال وصف وتحليل المواضيع التي تتشر من معمار والمطبخ المغربي واللباس التقليدي والحكايات الثعبية الخاصة بالمجتمع المغربي وذلك للوصول إلي نتائج تجيب علي تساؤلات البحث وتحقق أهدافه. 
بظهور الانترنت وسرعة انتشاره واستخدامه أكد هذا علي أهمية وسائل التواصل الاجتماعي في عملية تداول الاخبار والمعلومات ، ولقد زاد تأكيد أهمية تلك الوسائل من تعددها مما اتاح الفرصة للتواصل والتفاعل مع أكبر عدد ممكن من افراد المجتمع ، وتتميز وسائل التواصل الاجتماعي بقدرة كل فرد علي نشر ما بحوزته من معلومات وتفاعله مع ما ينشر بالتعليق والاضافة ، ولقد أصبحت وسائل التواصل الاجتماعي تمثل سمة مميزه لحياتتا اليومية وبات من المستحيل الاستغناء عنها ، حيث خلقت نوع جديد من التواصل الاجتماعي استطاع التغلب علي التواصل التقليدي بين الافراد ، فلقد قربت المسافات واتاحت معرفة مختلف ثقافات المجتمعات الأخرى من خلال التواصل مع تلك الشعوب والتعرف عليهح دون التقيد بالزمان والمكان ، ومع ذلك التطور السريع نتيجة التعرض المستمر لتلك وسائل التواصل الاجتماعي وزيادة حجم المعلومات التي يتلقها الفرد منها ومع انتشار الثقافات الغربية التي فرضت نفسها على الثقافات العربية فأصبح المجتمع العربي بصفة عامة والمجتمع المغربي بصفة خاصة يتأثر افراده بما يشاهدنه مما يؤثر هذا على قيمه وعادتهم التي قد توارثوها من أجدادهم ، فالمجتمع المغربي غني بالتراث الثقافي لذلك وجب الحفاظ عليه كونه رمز لهويتهم ، لذا تكمن مشكلة البحث في التعرف على كيفية استخدام وسائل التواصل الاجتماعي للحفاظ على التراث الثقافي للمجتمع المغربي.

$$
\text { يهدف هذا البحث التعرف علي : }
$$

- مدي استخدام وسائل التواصل الاجتماعي للحفاظ على التراث الثقافي المغربي. - وسائل التواصل الاجتماعي التي تتداول موضوعات ذات صلة بالتراث الثقافي المغربي. - أهم المواضيع المتداولة في وسائل التواصل الاجتماعي والمتضمنة التراث الثقافي المغربي.

3.1

يستمد البحث أهيته في كونه من الابحاث التي تتناول البعد الثقافي والاجتماعي في ظل التطور التكنولوجي عن طريق إبراز دور وسائل التواصل الاجتماعي فى التعرف على التراث الثقافي المغربي والحفاظ عليه وتوعيه المجتمع المغربي بأهميته وضرورة التمكك بهويته من خلال ما يتم نشره على تلك الوسائل من معلومات عن تراثهم العريق. 
- ما هي وسائل التواصل الاجتماعي التي تتداول موضوعات ذات صلة بالتراث الثقافي المغربي ؟

- - ما هي أهم المواضيع المتداولة فى وسائل التواصل الاجتماعي والمتضمنة التراث الثقافي المغربي ؟

ولقد ظهرت تلك النظرية خلال عقد السبعينيات من القرن الماضي كمنظور جديد لدراسة أثر وسائل الاعلام وخاصة التليفزيون وقامت النظرية على الجهود التي طورها الباحث الأمريكي "جورج جربنر" وزملاؤه في جامعة بنسلفنيا من خلال المشروع البحثي الخاص بالمؤشرات الثقافية والذى هدف إلى اقامة الدليل الامبيريقى على تأثير التليفزيون على البيئة الثقافية وأجرى هذا المشروع وفق ثلاث مراحل وهى : 1- فحص ودراسة السياسات الاعلامية الموجهة لتدفق المضمون الاتصالي • 2- تحليل دقيق ومفصل للبرامج والدراما التليفزيونية . 3- دراسة التأثيرات الناتجة عن التعرض المكثف لعالم التليفزيون • وخلص "جربنر" وزملأوه بأنه : كلما زاد الوقت الذى يقضيه الفرد أمام عالم التلفاز كلما كان إدراكه للواقع

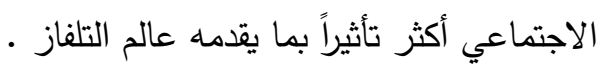
وتقوم النظرية على افتراض أن وسائل الاعلام تؤدى إلى خلق عادات مشتركة بين الافراد واختيار ومشاهدة الاحداث ويتم ذلك من خلال الرسائل التي تتتجها الوسائل وتوزعها بالاعتماد على التكنولوجيا الحديثة في الاتصال مما يؤدى إلى أساليب شائعة بين الافراد لرؤية العالم ، وكذلك فإن الرسائل الاعلامية المنظمة والمستمرة التي تعرضها وسائل الاعلام وخاصة التليفزيون تؤثر بشكل كبير على الجمهور (محسوب حجابي عبد الله ، 2007) 6.1 
أعتمد البحث على أداة تحليل المضمون حيث يقصد بكلمة تحليل تفكيك الكل إلي عناصر وأقسام مكونة له ، وكلمة مضمون يقصد بها ما يحتويه الوعاء اللغوي أو التصويري أو الإيمائي من معاني مختلفة فيقوم الفرد بالتعبير عنها في شكل رموز وفق تتظيم معين لتحقيق غايات اتصالية مع الأخرين. (عاطف عدلي العبد ، 2007) فتحليل المضمون أسلوب يستخدم قي تحليل محتوي المادة التي تقدمها وسائل الإعلام ، وذلك بالوصف الموضوعي المنظم الكمي للمحتوي الظاهر للوسيلة الاعلامية. (بسام عبد الرحمن مشاقبه ، 2010)

"هي منظومة من الثبكات الاكترونية التي تسمح المشارك فيها بإنثاء حساب خاص به ومن ثم ربطه من خلال نظام اجتماعي الكتروني مع أعضاء آخرين ، كذلك هي صفحات الويب التي يمكن أن تسهل التفاعل بين الأعضاء المشتركين في هذه الثبكة الاجتماعية الموجودة بالفعل علي الانترنت ، وتهدف إلي توفير مختلف وسائل الاهتمام والتي من شأنها تساعد علي التفاعل بين الأعضاء بعضهم وبعض ، ويمكن أن تثمل هذه المميزات : المراسلة الفورية ، الفيديو ، الدردشة ، تبادل الملفات ، مجموعة النقاش ... وغيرها". (أحمد عصام ، 2013) : 2.7.1

"هو جميع التظاهرات المادية والمعنوية التي تحمل في طياها بوادر ثقافية من معالم تاريخية (أعمال النحت والنقوش والآثار والتاريخ والفن..) وغيرها من التعابير المادية أو الغير مادية ذات قيمة ثقافية للإنسان". (بو غديري هشام ، 2015) 1.2 1.1.2 نثأة وسائل التواصل الاجتماعي مرت وسائل التواصل الاجتماعي بالعديد من المراحل والتطورات الكثيرة والسريعة في نفس الوقت ، فقد ظهرت أول هذه المواقع في الولايات المتحدة الامريكية ، حيث كانت تُتخدم للتواصل بين زُملاء الدراسة ، وأكثر ما تم الاهتمام به والتركيز عليه في تلك الآونة هو خدمة الرسائل ، وعلي الرغم من أن هذه المواقع قد وفرت الكثير من خدمات التواصل 
الراهنة إلا أنها فثلت في تحقيق الأرباح لمؤسسيها وانتهي الكثير منها بالفشل والاختفاء ، وسنذكر الأن أبرز هذه المواقع التي ظهرت في تلك الفترة :

1- موقع classmates: وهو أول مواقع التواصل التي كان الهدف منها الربط بين زملاء وأصدقاء الدراسة ، ويرجع تاريخ إنثاء هذا المواقع إلى سنة 1995م. 2- موقع sixdegrees: في عام 1997 تبع موقع (classmates) محاولة ناجحة أدت إلى ظهور موقع (sixdegrees) ، وقد أتاح هذا الموقع للأشخاص المتواصلين من خلاله العديد من الخدمات مثل : إرسال الرسائل ، إنثاء ملفات شخصية وطرح لمحات عامة عنهم فيها ، إضافة الاصدقاء والزملاء ، وعلي الرغم من ذلك انحسر الموقع وتم إغلاقه في سنه 2000م ، وذلك لعدم قدرته على تمويل الخدمات المقدمة من خلاله. 3- وتتبع هذه المواقع ظهور العديد من مواقع التواصل الاجتماعي الأخرى في الفترة من (1997م-1999م) مثل موقع (live journal) وموقع (cyworld) الذي تم تأسيسه في كوريا موقع الاسيويين الأمريكيين (asianavenue) موقع ذوي البشرة السمراء (blackplanet) وغيرها. 4- وخلال الفترة من (1999م-2001م) ظهر الكثير من المواقع ولكنها أيضاً لم تتجح مثل موقع (ryze) الذي كان مُختص بأصحاب الأعمال لتيسير التعاملات التجارية. 2.1.2 بدأ تطور وسائل التواصل الاجتماعي ونجاحه في عام 2003م بظهور العديد من المواقع مثل موقع (linkedin) وحصلت أيضاً انطلاقه كبيرة عند ظهور موقع ال facebook الذي انطلق رسمياً في عام 2004م حيث أتاح تكوين التطبيقات للمتطورين عام 2007م مما أدي إلى زيادة أعداد مُستخدميه بشكل كبير ، وقد كان موقع الفيسبوك الموقع الأشهر على الإطلاق من حيث عدد المستخدمين ، كما اعثُر الموقع الأول علي مستوي العالم. (مرام الحوامدة ، 2020) 3.1.2 1- فيسبوك Facebook : بدأ كثبكة اجتماعية في عام 2004م من قبل مؤسسه مارك زوكر بيرغ، وتابعت شركة فيسبوك النمو لتتحول إلى واحدة من كبرى الثركات التي تستحوذ على معظم تطبيقات التواصل الاجتماعي في العالم ، وهو موقع تواصل اجتماعي يسمح بمشاركة الصور والمنشورات والتعليقات والأخبار المحلية والعالمية ، بالإضافة إلى 
وجود الدردشة والألعاب وبث الفيديو المباشر ، وبحسب إحصائية سابقة ، وجد في نهاية عام 2018م على فيسبوك حوالي 2.32 مليار مستخدم في جميع أنحاء العالم •

2- مسنجر Messenger : تطبيق المحادثات الثهير ، والذي كان في البداية ميزة مراسلة ضمن تطبيق فيسبوك فقط ، إنما ومنذ عام 2011م جعل تطبيق مسنجر منفصل بذاته وأخذت ميزاته بالتوسع أكثر فأكثر بفضل ميزات وتحديثات مسنجر المتكررة يمكن للشركات الآن الإعلان عن منتجاتها وبرمجة روبوتات للاردشة مع العملاء ، وإرسال رسائل إخبارية والمزيد من التفاصيل على مسنجر ·

3- واتساب WhatsApp : تطبيق المراسلة الأكثر شهرة ، يستخدمه الناس في أكثر من 180 دولة ، استخدم واتساب في البداية كتطبيق للتواصل مع الأسرة والأصدقاء فقط ، وتدريجياً أصبح الناس يستخدونه للتواصل مع الشركات ، إذ استخدم الشركات الصغيرة تطبيق WhatsApp Business للتواصل ، بينما هنالك تطبيق WhatsApp خusiness API للشركات بالحصول على ملف تعريف أعمال مناسب ، والتواصل مع العملاء وتقديم الدعم لهم ، ومشاركة آخر التحديثات معهم حول مشترياتهم منها. 4- وي شات WeChat : على غرار الواتساب والمسنجر ، تطوّر وي شات من تطبيق مراسلة عادي إلى نظام أساسي شامل ، إذ بالإضافة إلى إمكانية إجراء المكالمات وإرسال الرسائل يمكن للمستخدم التسوق عبر الانترنت والدفع في وضع عدم الاتصال ، وإجراء الحجوزات وتحويل الأموال وغيرها ، حقيقةً نمت شعبية وي شات في الصين وبعض أجزاء من آسيا ، حيث يتم حظر بعض منصات التواصل الاجتماعي في تلك المناطق ، ويُعتبر وي شات بديلاً ممتازاُ للتطبيقات الأخرى في حال قمت بأعمال تجارية هنالك. 5- تويتر Twitter : تأسس على يد جاك دورسي ، وإيفان ويليامز ، ونوى غلاس ، ويمكن تعريفه بأنه موقع تواصل اجتماعي يتواصل فيه الأشخاص عبر رسائل قصيرة لا تتجاوز 115 حرف تسمى التغريدات ، كما تستطيع نشر الصور والفيديو، بالإضافة إلى أن معظم الثركات العالمية والثخصيات المؤثرة لديها حسابات رسمية على تويتر مثل 
6- لينكد ان Linkedln : وهو من وسائل التواصل الاجتماعي كما يعتبر كأكبر موقع للعلاقات المهنية في أنحاء العالم ، كما أنه أفضل وسيلة للتواصل بين الآخرين يستخدم لينكد إن في تبادل المعرفة ونشر الأفكار وعرض السير الذاتية والثهادات الموثوقة التي حصل عليها المستخدم ، كما أن العديد من الثركات الكبرى تستخدم الموقع في عرض فرص العمل ، تأسس في عام 2002م على يد كونستانتين جيريك ، وريد هوفمان ، وجان لوك فيلانت. 7- يوتيوب YouTube : تأسس في عام 2005م على يد جواد كريم ، وستيفن تشن ، وتثاد هارلي ، واشترته غوغل عام 2006م مقابل 1.65مليار دولار ، وهو عبارة عن موقع يقوم بعرض الفيديوهات المتتوعة في شتى المجالات ، ويعطي الفرصة لمستخدميه بالقيام بالبث المباشر ، كما أنه من أفضل المنصات لصناع المحتوي ، يمكنك إنثاء الحساب بثكل مجاني ، ورفع ما ترغب من فيديوهات على الموقع ، ولكن ذلك يقتضي الالتزام بأخلاقيات الموقع. 8- انستغرام Instagram : تأسس من قبل شركة فيسبوك في عام 2010م ، وهو أحد وسائل التواصل الاجتماعي المخصصة لالتقاط الصور والفيديو ومن ثم تعديلها ومشاركتها بعد أن ينشأ الثخص حساباً على الموقع ، كما أنه كل من لديه حساب على التطبيق سيتمكن من رؤية المنشورات والتفاعل معها ، وأضيف إليه مؤخراً ميزة المحادثات عبر الرسائل النصية والصوتية أيضاً. 9- ميديوم Medium : تأسس في عام 201م على يد أحد مؤسسي تويتر إيفان ويليامز ، وهو منصة التدوين الأولى لرواية القصص والتجارب الثخصية ، لمن لديهج تجارب في العديد من المجالات ومنها ريادة الأعمال والعمل الحر والإدارة ، وعلى عكس أغلب منصات التدوين العالمية ، يتم تصنيف المحتوى أو المدونات في الموقع بحسب معاير مختلفة، ومنها نسبة القراءة ومشاهدة المقال. 10- تيك توك TikTok : تيك توك هو شبكة اجتماعية لمشاركة مقاطع الفيديو الموسيقية القصيرة ، يقوم الناس وبخاصةً الثباب الصاعد، بتصوير فيديوهات قصيرة يقومون فيها بتحريك شفاههم تناغماً مع الأغنية التي يريدونها مع إدخال التعديلات (إذا أرادوا منهم) من يستخدمه أيضاً بغرض تعليمي كبرامج الطبخ وغيرها ، وأقصى مدة للتصوير هي 60 ثانية ، وكان تيك توك التطبيق الأكثر تتزيلاً في العالم في الربع الأول من عام 2018م ، حيث تغلُب وقتها على وسائل الاتصال الأخرى كالفيسبوك والاتستغرام ، واستحوذ تيك توك مؤخراً على تطبيق ميوزكلي Musically والذي هو شبكة اجتماعية لفيديوهات موسيقية مماثلة لتيك توك ، ولكن دُمجت معه مؤخراً ليصبح تيك توك ميوزكلي. 
11- فايبر Viber : يثبه فايير تطبيقات المراسلة الاجتماعية الرئيسية مثل واتساب أو مسنجر ، إذ يسمح بإرسال الرسائل والوسائط المتعددة ، والملصقات وملفات GIF ، والاتصال الاعتيادي كما يقدم العديد من الفرص للشركات ، مثلًا: في حال كنت رجل أعمال سيكون فايبر الحل الأمثل لك لثراء الإعلانات والترويج لعلامتك التجارية ، وعرض منتجاتك في قسم التسوق وتقديم الخدمات للعملاء.

12- سناب شات Snapchat : يركز تطبيق سناب شات على مشاركة الصور ومقاطع الفيديو القصيرة بين

الأصدقاء ، مع إمكانية إضافة فلاتر لطيفة ، وتحديد زمن (ثوان إذا أردت) يختفي فيه المقطع من جهاز المتلقي ، وهو ما جعل من إضافة القصة أمراً شائعاً ، والذي امتد وانتشر في النهاية على جميع وسائل التواصل الاجتماعي وأهمها الانستغرام ، إذ احتل انستغرام مكان سناب شات في ميزة إضافة القصص ، وأصبح سناب شات محط اهتمام المسوقين لعرض علاماتهم التجارية فقط. 13- بنترست Pinterest : على عكس معظم وسائل التواصل الاجتماعي ، فإن تركيز بنترست ليس الحصول على تفاعل أو التسلية إنما هي واحدة من المنصات الرائدة في العالم لتبادل الأفكار والحصول على الإلهام في مواضيع مختلفة يزور هذه المنصة أكثر من 200 مليون شخص كل شهر ، للحصول على منتجات جديدة ووصفات ، واكتثاف وجهات جديدة ، ومقالات وغيرها الكثير ليس ذلك فقط ، بل تعتبر بنترست فرصة كبيرة للشركات والعلامات التجارية التي تتطلع إلى بناء جمهور متفاعل وجذب زيارات قيمة إلى مواقعها على الويب. 14- تلغرام Telegram : يشبه التلغرام معظم تطبيقات التواصل الاجتماعي ، ويتميز بمدى أمانته وخصوصيته كتطبيق مراسلة ، تستقيد العلامات التجارية من تلغرام عن طريق برمجة روبوتات دردشة للنظام لتوفير الدعم للعملاء ، كما تستفيد من ميزة قناة التلغرام (Telegram Channel) ، والتي تبث عن طريقها الرسائل إلى عدد غير محدود من العملاء. 15- ريديت Reddit : هو موقع الإنترنت الرائد لنشر الأخبار عن مختلف المجالات ، يحتوي منشورات مختلفة ضمن تصنيفات متتوعة ، إذ يمكن للمستخدمين من خلاله إرسال أسئلة ومناقشتها ، وروابط وإدراج صور ، والتصويت أيضاً بالإيجاب أو الرفض. (معاوية زعرور ، 2020) 4.1.2 إيجابيات وسلبيات وسائل التواصل الاجتماعي 1.4.1.2 إيجابيات وسائل التواصل الاجتماعي 
1- تقليل الحواجز التي تعيق الاتصال: يُمكن استخدام مواقع التواصل الاجتماعي لنقل الأفكار والآراء المتعلقة بموضوع معين لعدد كبير من الأشخاص وبطريقة سهلة ، وذلك من أي مكان ، وفي أي وقت ، كما تساعد خاصية مشاركة الرأي المتاحة على وسائل التواصل الاجتماعي على فتح الأبواب لتبادل الآراء وتوسيع فرص المشاركة في التعبير عن الرأي. 2- توسيع دائرة العلاقات الاجتماعية: تعتبر شبكات التواصل الاجتماعي أداة مفيدة وفعالة في تثكيل أصدقاء جُدد ، وتسهيل التواصل مع الأصدقاء الذين انقطع الاتصال بهم ، أو مع الأشخاص الذين لا يمكن مقابلتهم شخصياً، مما يوفر عناء الوصول إليهم. 3- وسيلة فعالة للترويج: تستخدم الشركات التجارية الشبكات الاجتماعية كأداة جيدة من أجل الترويج لسلعها، حيث يوجد العديد من التطبيقات الكُختصة بالترويج لخدمة أو سلعة معينة وبتكلفة أقل، مما يؤدي إلى زيادة الأرباح وبأقل التكاليف. 4- وسيلة لتشكيل رأي عام فعال: تُعد مواقع التواصل الاجتماعي بما تؤمنه من تفاعل واسع بين الهجوعات وسيلة لتثكيل رأي عام مساند لبعض القضايا، وهو الأمر الذي ينتج عنه تغيير إيجابي في بعض مناحي الحياة. 5- متابعة الأخبار: أدى تطور شبكات التواصل الاجتماعي إلى عدم انتظار الثخص أخبار الساعة السادة على شاشة التلفاز، أو انتظار وصول الجريدة، بحيث يمكن معرفة آخر الأخبار والمعلومات من خلال وسائل التواصل الاجتماعي. 6- مساعدة رجال الأعمال والثركات: تُككن شبكات التواصل الاجتماعي رجال الأعمال والمنظمات الدختلفة من التواصل مع العملاء ، وبيع منتجاتهم ، وتوسيع نطاق خدماتهم ، فهناك الكثير من رجال الأعمال والثركات التي تزدهر بشكل كامل على الشبكات الاجتماعية ، ولا تكون قادرة على العمل بدونها. 2.4.1.2 سلبيات وسائل التواصل الاجتماعي 1- مخاطر الاحتيال أو سرقة الهوية: يمكن الوصول إلى المعومات الخاصة التي تُشر على الإنترنت من أي شخص ، حيث يكون كل ما يحتاج إليه حينها عدد قليل من المعلومات للتأثير على حياة الثخص ، كما يتضمن هذا الخطر اختراق المعلومات الثخصية والتطفل عليها. 
2- ارتكاب الجرائم ضد المستخدمين: يمكن أن يؤدي استخدام الثبكات الاجتماعية إلى تعرض الأشخاص

للمضايقات بكافة أشكالها ، وقد يكون هذا شائعاً خاصةً لدى المراهقين والأطفال الأصغر سناً بشكل خاص، لذا ينبغي على الوالدين الانتباه لمحتوى الويب الخاص بهج، حتى لا يتعرض الأطفال لأي محتوى غير مناسب.

3- التأثير على العلاقات الأسرية: تلعب مواقع التواصل الاجتماعي دوراً سلبياً في نوعية العلاقات الأسرية وقوتها، حيث يؤدي ما يقضيه الفرد من ساعات طويلة في تصفح هذه المواقع، وانشغاله بعلاقاته الافتراضية فيها إلى البعد عن أفراد أسرته وفتور العلاقات التي تربطه بهم.

4- مخالفة منظومة العادات والتقاليد: قد يؤدي الانفتاح الزائد الذي تؤمنه هذه المواقع إلى نشر قيم جديدة مخالفة

$$
\text { لما اعتاد عليه المجتمع من عادات وتقاليد تشكل هويته. (إبراهيم العبيدي ، 2020) }
$$

1.3

1.1.3 عناصر التراث الثقافي

حدد اليونسكو العناصر التالية :

1- الآثار : وتثمل كل الأعمال المعمارية وأعمال النحت والتصوير على المباني، وكل العناصر والتكوينات ذات الصفة الأثرية، والنقوش والكهوف، ومجموعات المعالم التي لها قيمة عالمية استثنائية من وجهة نظر التاريخ

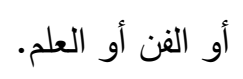

2- المجمعات: مجموعات المباني المنعزلة أو المتصلة، التي لها بسبب عمارتها أو تتاسقها أو اندماجها في منظر طبيعي قيمة عالمية استثائية من وجهة نظر التاريخ أو الفن أو العلم. 
3- المواقع: أعمال الإنسان أو الأعمال المشتركة بين الإنسان والطبيعة ، وأيضا المناطق بما فيها المواقع الأثرية

$$
\text { التي لها قيمة استثنائية من الوجهة التاريخية أو الجمالية، أو الإثتولوجيا أو الأنثروبولوجيا. }
$$

وينظر إلى التراث الثقافي أيضا بأنه: شكل ثقافي متميز يعكس الخصائص البشرية عميقة الجذور، وتتناقله الأجيال المتعاقبة، ويصمد عبر فترات زمنية متفاوتة نوعياً ومتميزة بيئياً ، وتظهر عليه التغيرات الثقافية الداخلية والعادية، ولكنه يحتفظ دائما بوحدة أساسية مستمرة.

ويرى آخرون بأن التراث يمثل مجموع قيم ومعتقدات وآداب وفنون، ومعارف تثمل جميع أنثطة الإنسان المادية والمعنوية الناتجة عن تراكم خبرات المجتمع، وهو بذلك شاهد على تاريخ الأمم وأحوالها، ويتميز بأنه يتكون من بنى مترابطة ومتكاملة الأجزاء ومتداخلة فيما بينها في أغلب الأحيان، ومنه مـا هـو ثابت ومنه ما هو متغير . ونخلص بأن التراث يمثل هوية الثعوب الأمر الذي يستوجب التمسك بأصالته والمحافظة عليه، فهو التاريخ المادي والمرآة الحقيقية لأي حضارة ، وهكذا تبقى علاقة الإنسان بتراثه علاقة عضوية تتمثل في هويته وترتبط بمدى وعيه بأبعاد حضارية وتاريخية ودينية وثقافية واجتماعية وسياسية على حد سواء.

\subsection{3 أقسام التراث الثقافي:}

يتكون التراث الثقافي من عناصر غير منقولة: مثل المواقع والمعالم والمباني الأثرية، والتاريخية، ومنقولة: مثل القطع الأثرية، وقطع التراث الثعبي، والحرف اليدوية، وأيضا من عناصر التراث غير المادي، مثل العادات والفنون الثعبية. ويعد التراث الثقافي جزءاً لا يتجزأ من الهوية الوطنية ، لذا فإن فقدان أي من عناصره ، يعد فقدان لجزء من هذه الهوية ، وخسارة لقيم متميزة لا تقدر بقيمة ، فإن التراث الثقافي يقسم عادة إلى قسمين رئيسيين، وهما:

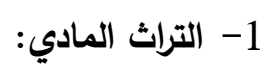

يشمل المباني والأماكن التاريخية والآثار والتحف، وما تكثفه الحفريات وتضمه المتاحف، وكل منها يمثل فترات تاريخية في حياة الثعوب ، الأمر الذي يستوجب حمايتها والحفاظ عليها بثكل مستدام لأجيال المستقبل وهي بذلك بمثابة عناصر متميزة بالنسبة لعلم الآثار والهندسة المعمارية والعلوم أو التكنولوجيا ، سيما ما يرتبط منها بالعناصر الثقافية وتصبح تلك المكونات ذات أهمية لدراسة تاريخ البشرية لأنها تمثل الركيزة الأساسية لأفكار على مر الزمن.

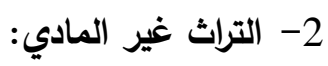


توسع مفهوم التراث الثقافي ولم يعد يقتصر على المعالم التاريخية ومجموعات القطع الفنية والأثرية ، وإنما أصبح يشمل أيضا التقاليد أو أشكال التعبير الحية الموروثة عن السلف ، والتي تتداولها الأجيال على مر الزمن مثل: التقاليد الثفهية والفنون الاستعراضية والممارسات الاجتماعية والطقوس والمناسبات الاحتفالية والمعارف والممارسات المتعلقة بطبيعة الكون والمعارف والمهارات المستخدمة في الصناعات الحرفية التقليدية ، وهذا ما يعرف بالتراث الثقافي غير المادي ، ويُقصد بالتراث الثقافي غير المادي وفقاً للتعريف الوارد في "اتفاقية صون التراث الثقافي غير المادي" لليونسكو بأنه: "مجمل الإبداعات الثقافية، التقليدية والثعبية، المنبثقة عن جماعة ما والمنقولة عبر التقاليد، ومنها: اللغات والقصص والحكايات، والموسيقى والرقص وفنون الرياضة التتالية، والمهرجانات والطب وحتى فن الطهي. (صليحة عشي ، 2011) 3.1.3 أنواع التراث المغربي

ـ التراث الثفوي (تراث غير مادى): وهو تراكم خبرة الانسان عبر أجيال لذلك فعلاقة الانسان به علاقة عضوية فهو الذاكرة الحية للفرد والمجتمع ، كما أن لكل شعب موروثاته الخاصة به والتي يتوارثها شفهياً ، لذلك فهو إنتاج جماعي يختزن خبرات الافراد والجماعات ، ويعد التراث الثفوي جدار متين لحفظ هويتهم ومحرك لها في الاستمرارية والوجود. - التراث المكتوب: وهو الوثائق والمخطوطات والنصوص التاريخية ...

- التراث المبني : يوجد بالمغرب خمس مدن مصنفة عالميأ في قائمة التراث العالمي باليونسكو وهى : مراكش ، وفاس ، ومكناس ، وتطوان ، والصويرة. المساجد والمدارس : جامع القرويين ومدرسة العطارين بمدينة فاس الابواب والقصبات : باب منصور لعلج بمكناس وباب أكناو بمراكش - التراث المنقول : يوجد بالمغرب قطع أثرية متعددة ومتتوعة (نقود ووثائق وأوان خزفية) محفوظة بمختلف المتاحف المغربية والدولية ومنها متحف الاسلحة بفاس ومتحف الاثار بمدينة الرباط ودار السي سعيد بمراكش ومتحف الفنون الصحراوية بالعيون. - مواقع أركيولوجية : وهو الدكان الذي يضم الدليل الاثري ، وتعود المواقع الاثرية لفترة ما قبل التاريخ وتوجد مجموعة من هذه المواقع بسلا دار بوعزة بالدار البيضاء وتافوغالت بالثرق ومزورة بالشمال ، ويوجد مواقع أثرية للعهد الفينيقي والروماني من بينها موقع وليلي المسل ضمن التراث العالمي منذ 1977. (almawadia3.blogspot.com/2018) 
1.4 نماذج من وسائل التواصل الاجتماعي التي تتناول موضوعات عن التراث الثقافي المغربي

مع مرور السنين نلاحظ اندثار الكثير من تراثثا الثقافي ، بسبب عدم القدرة على نشره بين الاجيال ومع التطور التكنولوجي والذي سهل تلك العملية من خلا توفير مساحة للتواصل والحوار ، فبانتشار التقنيات الحديثة من وسائل التواصل الاجتماعي أصبح من السهل الاستفادة منها وتطويعها في الحفاظ علي التراث الثقافي المغربي والعمل علي نشره بين أفراد المجتمع بطريقة سهلة وجذابة في الثكل والمضمون ، لذا فإن تلك الوسائل تلعب دور فعال في نشر تراثها الثقافي ، ومن بين تلك الوسائل صفحات الفيسبوك وقنوات اليوتيوب لما لهم من جمهور كبير من المستخدمين لما يوفرونه من خدمات يسهل استخدامهم والتي تميزهم عن باقي الوسائل في عملية تبادل الموضوعات وتتوعها ، وسيتم عرض بعض النماذج لتلك الوسائل ومنها:

"Morocco 1.1 .4

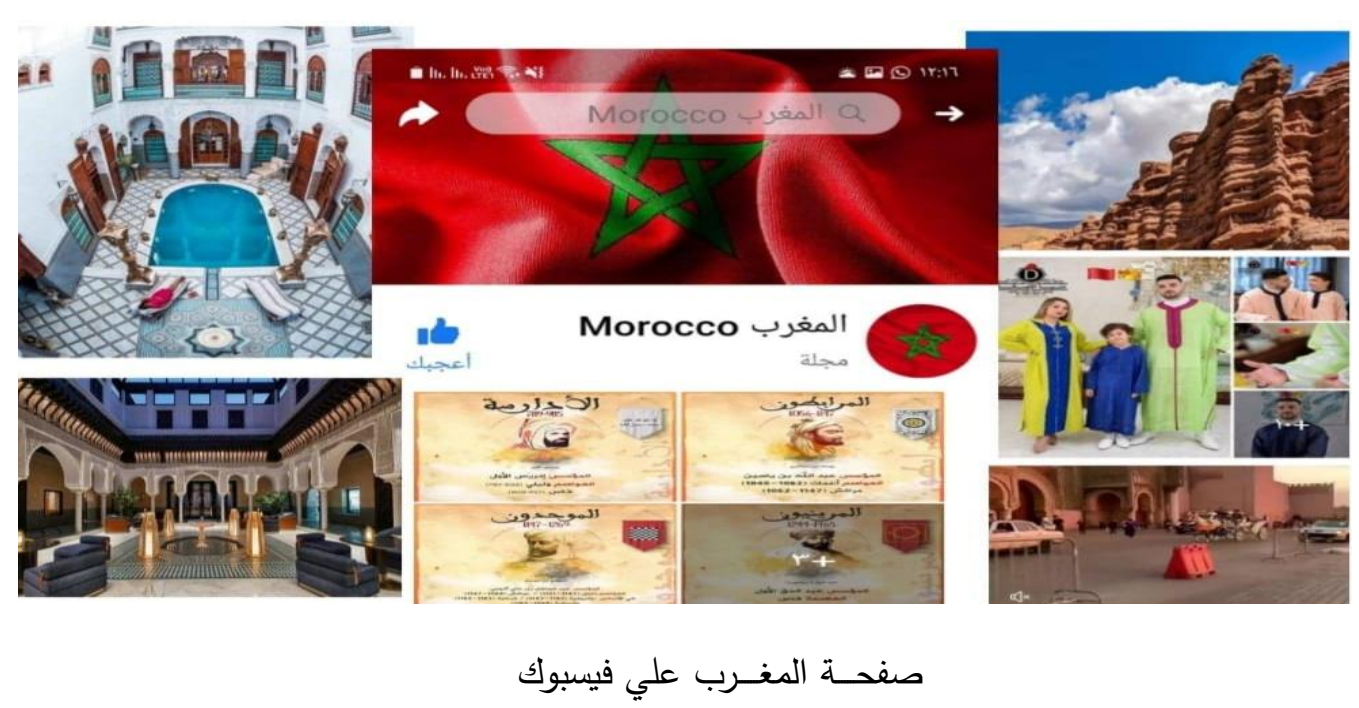

وتتناول صفحة "الدغرب Morocco" موضوعات متتوعة ومتعددة عن التراث بالمغرب ، عدد معجبيها يصل إلي

24 الف معجب وعدد متابعيها 28 الف متابع ، تهتم الصفحة بعرض متتوع عن التراث الثقافي سواء كان مادي أو اللامادي وتسعي الصفحة لإبراز ذلك التتوع والتي تزخر به المغرب ، فاهتمام الصفحة هو الجانب السياحي للمغرب من أجل المساهمة في التعريف بها والترويج لها علي المستويين الداخلي والخارجي من خلال عرض صور وفيديوهات تُعرف مستخدميها بما تملكه المغرب من مواقع اثريه مهمه ومعمارية تتميز بها ، وكذلك تعرض صور للباس التقليدي لهم وتاريخ سلاطينهم ، ويصاحب الفيديوهات التي يتم عرضها عن مواقع وموضوعات خاصة بالمغرب صوت يمتاز باللهجة المغربية 
، وبمشاهدة الصفحة نلاحظ أن الهدف منها هو تعريف الافراد بالتراث المغربي والمساهمة في توعيتهم وتثقيفهم بهويتهم لضمان الحفاظ عليها وانتقالها من جيل إلى جيل ، ونجد من تعليقات زائري الصفحة مدي وعيهم وإدراكهم و واستجابتهم لذلك. (www.facebook.com/Morocco) 2.1.4 قناتي اليوتيوب "الفراشة" و"عالم الأناقة والجمال المغربي"

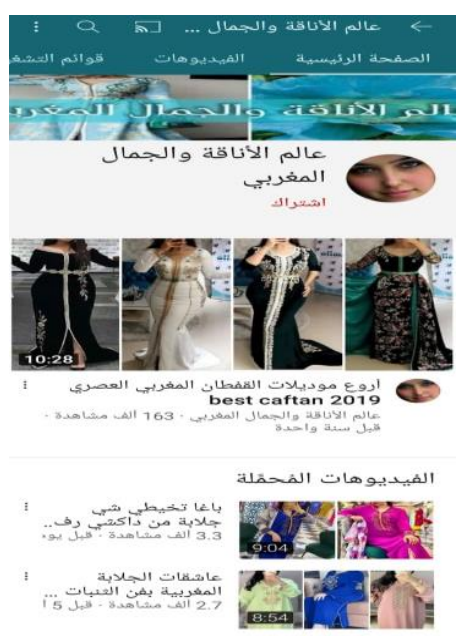

قناة عالم الأناقة والجمال المغربي

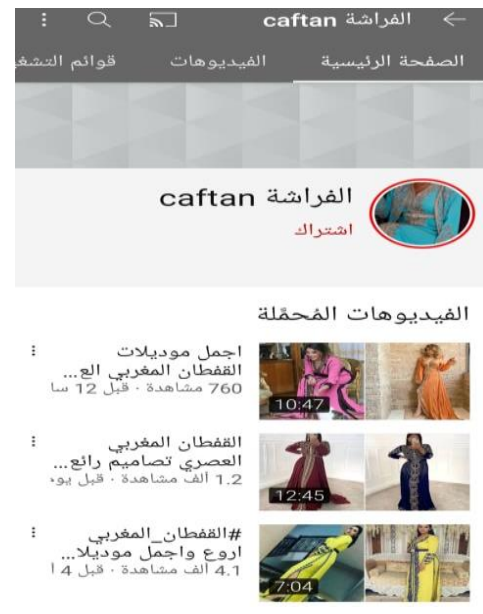

قناة الفراشة

تعد كلاً من قناة "الفراشة" وقناة "عالم الأناقة والجمال المغربي" قناتين تهتمان بموضوعات مختصة بالمرأة من حيث اللباس المغربي العصري والتقليدي من قفطان وراندة وجلابه ، يصل عدد مشاهدي قناة الفراشة إلى 800 الف مشاهد أما مشاهدي قناة عالم الأناقة والجمال المغربي يصل إلى 11 مليون مشاهد ، تهتم القناتين بعرض التراث الثقافي اللامادي عن طريق إبراز اللباس التقليدي لهم ، والتي تزخر به المغرب من خلال عرض فيديوهات تُعرف مشاهديهم بما تملكه المغرب من لباس تقليدي مختص بالمرأة ، ويصاحب الفيديوهات التي يتم عرضها صوت يمتاز باللهجة المغربية ، وبمشاهدة القنوات نلاحظ أن الهدف منها هو تعريف الافراد باللباس التقليدي للمغرب وذلك للمساهمة في توعيتهم وتثقيفهم بهويتهم لضمان الحفاظ عليها وانتقالها من جيل إلى جيل ، ونجد من تعليقات مشاهدي القناتين مدي وعيهم واستجابتهم لذلك. (www.youtube.com/channel/(UCJnufyDNCFowdWeTNLPDzHg),( UCBGbAX63Rcth1F4oE)) 3.1 .4

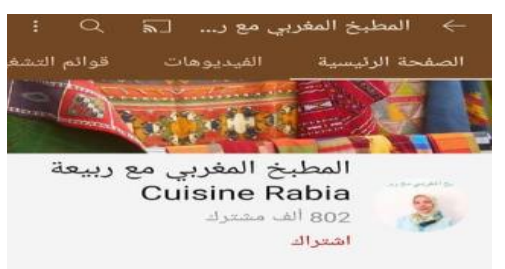




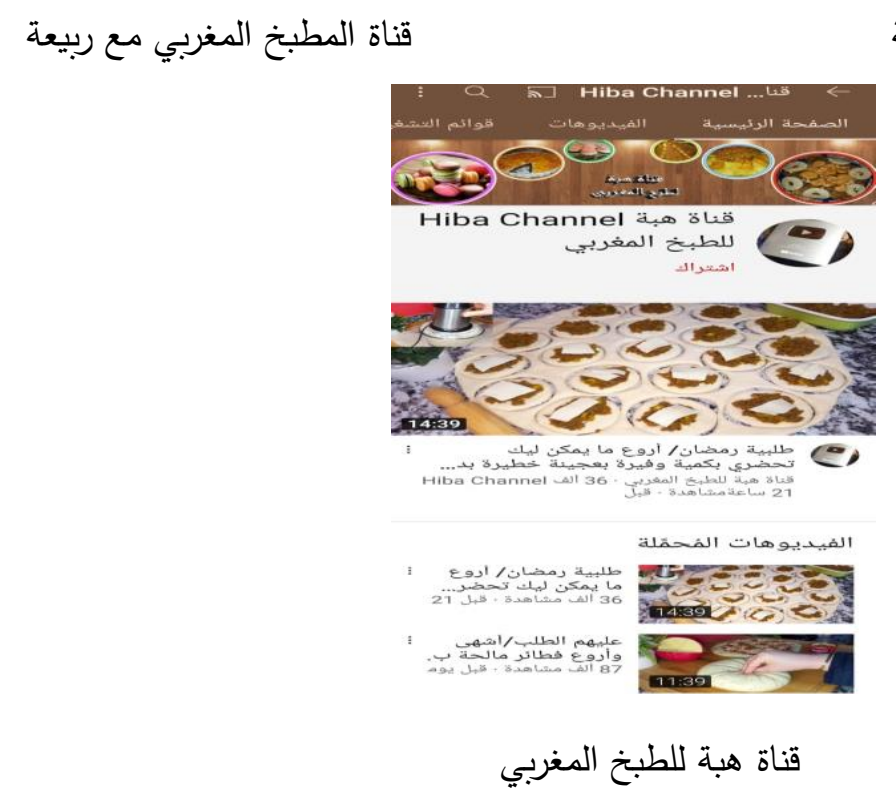

قناة نكهة مغربية

تعد كلاً من قناة "نكهة مغربية" و"المطبخ المغربي مع ربيعة" و"هبة للطبخ المغربي" قنوات مختصة بالمطبخ

المغربي من طبخ طواجن ومعجنات وحلويات ومشروبات ، يصل عدد مشاهدي قناة نكهة مغربية إلى 29 مليون مشاهد أما مشاهدي قناة المطبخ المغربي مع ربيعة يصل إلى 136 مليون مشاهد ومشاهدي قناة هبة للطبخ المغربي يصل إلى 118 مليون مشاهد ، تهتم القنوات بعرض التراث الثقافي اللامادي كما تسعي لإبراز المطبخ الدغربي التقليدي الذي يزخر به المغرب بأصنافه المتعددة من الأطعمة عن طريق عرض فيديوهات تُعرف مشاهديهم بما تملكه المغرب من أكلات تقليدية ، ويصاحب الفيديوهات التي يتم عرضها صوت يمتاز باللهجة المغربية ، وبمشاهدة القنوات نلاحظ أن الههف منها هو تعريف الافراد بالأكلات التقليدية للمغرب من خلال عرض عادات وتقاليد المجتمع المغربي من مواسم وأعياد وما يتم إعداده بالمطبخ المغربي في تلك المناسبات ، وذلك للمساهمة في توعيتهم وتثقيفهم بهويتهم لضمان عدم اندثارها والحفاظ 
عليها وانتقالها من جيل إلى جيل ، ونجد من تعليقات مشاهدي القنوات مدي وعيهم واستجابتهم لذلك للحفاظ علي عاداتهم (www.youtube.com(user/cokimaroc),( UCFldnUF7Y4JbUf7C),( UCDxusNQSUK4XoDwL3)). وتقاليد (wم)

\section{1 .4 قناتسي اليوتيوب "حجايات شعبية باللسان المغربي الدارج" و"حكايات شعبية ما /حلاها"}

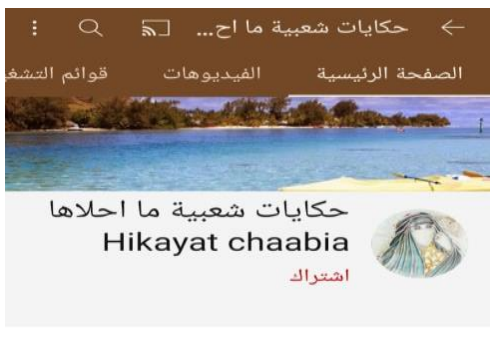

الفيبيوهات المحمُلة

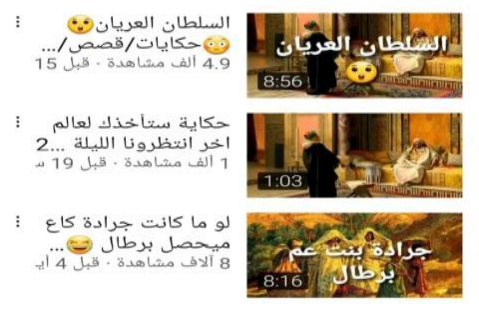

قناة حكايات شعبية ما احلاها

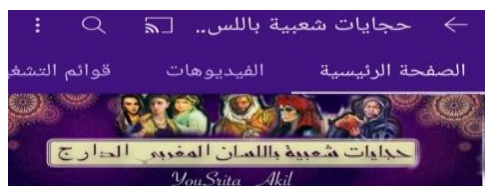

حجايات شعبية باللسان

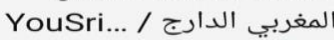

$$
\text { اشتراك المغربي }
$$

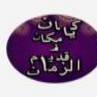

الفيبيوهات المُحمًّلة

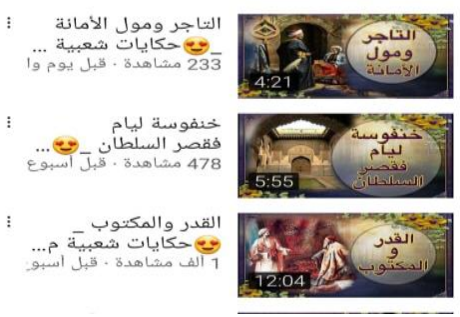

قناة حجايات شعبية باللسان المغربي الدارج

تعد كلاً من قناة "حجايات شعبية باللسان المغربي الدارج" و"حكايات شعبية ما احلاها" قنوات مختصة بالحكايات الثعبية المغربية ، يصل عدد مشاهدي قناة حجايات شعبية باللسان المغربي الدارج إلى 760 الف مشاهد أما مشاهدي قناة حكايات شعبية ما احلاها يصل إلى 3 مليون مشاهد ، تهتم القناتين بعرض التراث الثقافي اللامادي وتسعي القناتين لإبراز القصص والحكايات الثعبية العربية بصفة عامة والمغربية بصفة خاصة ، والذي يزخر به المغرب من خلال عرض فيديوهات تُعرف مشاهديهم بالتراث الثعبي المغربي ، وذلك للمساهمة في توعيتهم وتثقيفهم بهويتهم لضمان عدم اندثارها وتبليغه للأجيال القادمة في عالم يتجه نحو الرقمية بطريقة متوحشة ، ويصاحب الفيديوهات التي يتم عرضها صوت يمتاز باللهجة المغربية ، ونلاحظ من تعليقات مشاهدي القناتين مدي وعيهم واستجابتهم لذلك من أجل الحفاظ علي تراثهم. (www.youtube.com/channel/(UCrrD9ia9p0QGwi6Ul0nys5g),( UCoUkqGmD8Wy5TxjN7Z6o8FA))

$$
1.5
$$

$$
\text { توصل البحث إلى عدد من النتائج تمثلت في : }
$$


1- تلاحظ مدي اهتمام ووعي المجتمع المغربي باستخدام وسائل التواصل الاجتماعي من أجل نشر تراثه الثقافي عليها ، وذلك للحفاظ عليه من الاندثار والتلاشي عن طريق تبليغه من جيل إلي جيل عبر تلك الوسائل لما لها من أهمية في عصرنا اليوم.

2- كان من أهم وأبرز وسائل التواصل الاجتماعي والتي أهتمت بنشر التراث الثقافي المغربي ، وتم التطرق إليها في هذا البحث هي "الفيسبوك" و "اليوتيوب" ، لما يمتلكونه من أدوات يسهل استخدامها في أي وقت ومكان عن طريق ما يوفرونه من صور وفيديوهات ومنشورات وتعليقات ، والتي تجعل مستخدميهم علي تفاعل مستمر مع تلك الصفحات والتنوات ، ومعرفة ما تقدمه تلك الوسائل من تراث ثقافي سواء كان مادي أو اللامادي يخص المجتمع المغربي. 3- تضمنت صفحة فيسبوك "المغرب" ، وقنوات يوتيوب: "الفراشة" ، "عالم الأناقة والجمال المغربي" ، "نكهة مغربية" ، "المطبخ المغربي مع ربيعة" ، "قناة هبة للطبخ المغربي" ، "حجايات شعبية باللسان المغربي الدارج" ، "حكايات شعبية ما احلاها" مجموعة من المعلومات والأفكار حول التراث الثقافي المادي واللامادي من عرض لمناطق أثرية ومعمارية بالمغرب ، كذلك عرض اللباس التقليدي للمجتمع المغربي وعرض المطبخ المغربي بأكلاته التي يمتاز بها بالإضافة إلى عرض حكايات من التزاث الثعبي المغربي مبرزه بذلك مدي التتوع في التعبير عن تراثها الثقافي محاوله بذلك نشر فكره الحفاظ على تراثها الثقافي من محاولة الاندثار . ومما تم عرضه يتضح لنا أن لوسائل التواصل الاجتماعي في عصرنا الحديث أهمية فلقد أصبحت عباره عن وسائل توثيقية من خلال ما ينشر بها من موضوعات ذات صلة بالتراث الثقافي المغربي ، وذلك للعمل على استمراره للأجيال القادمة حتي لا تطغي عليه الثقافات الغربية. 
1- ضرورة انثاء المزيد من صفحات الفيسبوك وقنوات اليوتيوب والمتخصصة في مجال التراث الثقافي

$$
\text { المغربي. }
$$

2- على الجهات الحكومية والجهات الخاصة المعنين بالتراث الثقافي المغربي التعاون من أجل انثاء مواقع

وصفحات وقنوات مدفوعة الاجر حتي يكون لها صدي وانتشار سواء كان ذلك داخل المجتمع المغربي أو

خارجه من أجل التعرف بتراثهم والحفاظ على هويتهم. 
المراجع

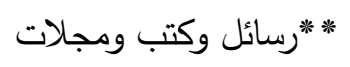

1- أحمد عصام (2013) : استخدام الثباب الجزائري لوسائل التواصل الاجتماعي ، رسالة ماجستير ، كلية العلوم الانسانية والاجتماعية ، قسم علوم الاعلام والاتصال ، جامعة المسيلة ، الجزائر ، ص23،ص24. 2- بسام عبد الرحمن مشاقبه (2010) : مناهج البحث الاعلامي وتحليل الخطاب ، دار أسامة للنشر والتوزيع ، عمان ،

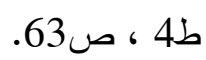
3- بو غديري هثام (2015) : الحماية الدولية للتراث الثقافي والطبيعي ، رسالة ماجستير ، كلية الحقوق ، جامعة

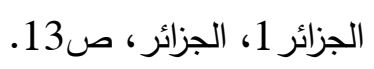

4- صليحة عشي (ديسمبر 2011) : صيانة الموروث الثقافي في الجزائر كجزء من مقومات التتمية السياحية المستدامة ، ندوة الدولية الأولى للعمران والسياحة المستدامة، جامعة المسيلة، الجزائر ، ص665. 5- عاطف عدلي العبد (2007) : بحوث الاعلام والرأي العام تصميمها وتتفيذها ، دار الفكر العربي ، القاهرة ، ط4 .46، 6- محسوب حجابي عبد الله (2007) : دور وسائل الاعلام في تكوين الصورة الذهنية عن الصعيد لدى الثباب الدصري ، رسالة ماجستير ، كلية الاعلام، جامعة القاهرة ، ص38، *** الانترنت * 1- إبراهيم العبيدي (6ديسمبر 2020) : سلبيات وإيجابيات مواقع التواصل الاجتماعي : https://mawdoo3.com/\%D8\%B3\%D9\%84\%D8\%A8\%D9\%8A\%D8\%A7\%D8\%AA_\%D9 \%88\%D8\%A5\%D9\%8A\%D8\%AC\%D8\%A7\%D8\%A8\%D9\%8A\%D8\%A7\%D8\%AA_\%D9 \%85\%D9\%88\%D8\%A7\%D9\%82\%D8\%B9_\%D8\%A7\%D9\%84\%D8\%AA\%D9\%88\%D8\% A7\%D8\%B5\%D9\%84_\%D8\%A7\%D9\%84\%D8\%A7\%D8\%AC\%D8\%AA\%D9\%85\%D8\%A 7\%D8\%B9\%D9\%8A

$$
\text { 2- مرام الحوامدة (28 فبراير 2020) : ما هي وسائل التواصل الاجتماعي }
$$


https://e3arabi.com/\%D8\%A7\%D9\%84\%D8\%AA\%D9\%82\%D9\%86\%D9\%8A\%D8\%A9/ \%D9\%85\%D9\%88\%D8\%A7\%D9\%82\%D8\%B9\%D8\%A7\%D9\%84\%D8\%AA\%D9\%88\%D 8\%A7\%D8\%B5\%D9\%84\%D8\%A7\%D9\%84\%D8\%A7\%D8\%AC\%D8\%AA\%D9\%85\%D8 \%A7\%D8\%B9\%D9\%8A/

$$
\text { 3- معاوية زعرور (3 نوفمبر 2020) : ما هي وسائل التواصل الاجتماعي واهميتها واشهرها }
$$

https://www.arageek.com/l/\%D9\%85\%D8\%A7-\%D9\%87\%D9\%8A\%D9\%88\%D8\%B3\%D8\%A7\%D8\%A6\%D9\%84\%D8\%A7\%D9\%84\%D8\%AA\%D9\%88\%D8\% A7\%D8\%B5\%D9\%84\%D8\%A7\%D9\%84\%D8\%A7\%D8\%AC\%D8\%AA\%D9\%85\%D8\%A7\%D $8 \% B 9 \% \mathrm{D} 9 \% 8 \mathrm{~A}$

4- http://almawadia3.blogspot.com/2018/04/blog-post_89.html

5- https://www.facebook.com/Morocco.maroc.marocaine

6- https://www.youtube.com/channel/UCJnufyDNCFowdWeTNLPDzHg/featured

7- https://www.youtube.com/channel/UCBGbAX63Rcth1F4oE-GafoA

8- https://www.youtube.com/user/cokimaroc

9- https://www.youtube.com/channel/UCFIdnUF7Y4JbUf7C-wkwSEA

10- https://www.youtube.com/channel/UCDxusNQSUK4XoDwL3ITiWqw

11- https://www.youtube.com/channel/UCrrD9ia9p0QGwi6Ul0nys5g

12- https://www.youtube.com/channel/UCoUkqGmD8Wy5TxjN7Z608FA 